\title{
A theory-based process evaluation alongside a randomised controlled trial of printed educational messages to increase primary care physicians' prescription of thiazide diuretics for hypertension [ISRCTN72772651]
}

Justin Presseau, ${ }^{1,2}$, Jeremy M. Grimshaw ${ }^{1,3^{*}}$, Jacqueline M. Tetroe ${ }^{4}$, Martin P. Eccles 5 , Jill J. Francis ${ }^{6}$, Gaston Godin? Ian D. Graham ${ }^{1,2,8}$, Janet E. Hux ${ }^{9}$, Marie Johnston ${ }^{10}$, France Légaré ${ }^{11}$, Louise Lemyre ${ }^{12}$, Nicole Robinson ${ }^{1}$ and Merrick Zwarenstein ${ }^{13,14}$

\begin{abstract}
Background: Pragmatic trials of implementation interventions focus on evaluating whether an intervention changes professional behaviour under real-world conditions rather than investigating the mechanism through which change occurs. Theory-based process evaluations conducted alongside pragmatic randomised trials address this by assessing whether the intervention changes theoretical constructs proposed to mediate change. The Ontario Printed Educational Materials (PEM) cluster trial was designed to increase family physicians' guidelinerecommended prescription of thiazide diuretics. The trial found no intervention effect. Using the theory of planned behaviour (TPB), we hypothesised that changes in thiazide prescribing would be reflected in changes in intention, consistent with changes in attitude and subjective norm, with no change to their perceived behavioural control (PBC), and tested this alongside the RCT.

Methods: We developed and sent TPB postal questionnaires to a random sub-sample of family physicians in each trial arm 2 months before and 6 months after dissemination of the PEMs. We used analysis of covariance to test for group differences using a $2 \times 3$ factorial design. We content-analysed an open-ended question about perceived barriers to thiazide prescription. Using control group data, we tested whether baseline measures of TPB constructs predicted self-reported thiazide prescribing at follow-up.

(Continued on next page)
\end{abstract}

\footnotetext{
* Correspondence: jgrimshaw@ohri.ca

1 Ottawa Hospital Research Institute, The Ottawa Hospital, General Campus,

501 Smyth Road, Box 201B, Ottawa, Ontario K1H 8L6, Canada

${ }^{3}$ Department of Medicine, University of Ottawa, 451 Smyth Rd, Ottawa,

Ontario K1H 8M5, Canada

Full list of author information is available at the end of the article
} 
(Continued from previous page)

Results: Four hundred twenty-six physicians completed pre- and post-intervention questionnaires. Baseline scores on measures of TPB constructs were high: intention mean $=5.9$ out of $7(S D=1.4)$, attitude mean $=5.8(S D=1.1)$, subjective norm mean $=5.8(S D=1.1)$ and PBC mean $=6.2(S D=1.0)$. The arms did not significantly differ postintervention on any of the theory-based constructs, suggesting a possible ceiling effect. Content analysis of perceived barriers suggested post-intentional barriers to prescribing thiazides most often focused on specific patient clinical characteristics and potential side effects. Baseline intention $(\beta=0.63, p<0.01)$ but not PBC ( $\beta=0.04$, $p=0.78$ ) predicted $42.6 \%$ of the variance in self-reported behaviour at follow-up in the control group.

Conclusions: Congruent with the Ontario Printed Educational Messages trial results and aligned with the TPB, we saw no impact of the intervention on any TPB constructs. The theoretical basis of this evaluation suggests possible explanations for the failure of the PEM intervention to change professional behaviour, which can directly inform the design and content of future theory-based PEM interventions to change professional behaviour.

Trial registration: ISRCTN, Canada ISRCTN72772651

\section{Background}

Hypertension is widespread. In the province of Ontario in Canada, $21 \%$ of the adult population has an elevated blood pressure, with prevalence rising as a function of age to $52 \%$ in those aged 60-79 [1]. Rates in Ontario are largely consistent with prevalence rates across Canada as a whole [2]. While numerous hypertension medication options are available for managing hypertension, thiazide diuretics are among the most well tolerated [3], have cardiovascular protective effects [4] and have been consistently recommended as first-line agents in clinical practice guidelines for managing uncomplicated hypertension $[5,6]$. They are also the least expensive and, if more widely used, would result in substantial annual savings if used over more expensive drug options [7]. In spite of this, thiazides are not prescribed as often as other antihypertensive drugs [8].

Reviews of disseminating printed educational materials (PEMs) suggest that they can be effective in promoting health professional behaviour change in some instances but not in all, and there is wide variation in effectiveness and methodological rigour between existing randomised controlled trials (RCTs) [9, 10]. The large factorial cluster randomised Ontario Printed Educational Messages (OPEM) trial (and associated TRY-ME sub-trial) for promoting thiazide diuretic prescription was designed to address the limitations of previous trials [11]. This trial tested the effectiveness of short and long educational messages (in this case, PEMs) for increasing thiazide prescription to elderly patients with uncomplicated hypertension, a recommended antihypertensive drug that is at least as effective as other classes of antihypertensive at reducing morbidity and mortality while being less expensive. The trial found no evidence that PEMs increased the number of patients receiving thiazide diuretics. While the size and rigour of the trial provide convincing evidence that the PEMs were not effective for changing this clinical behaviour, the trial was not designed to investigate the reason for this lack of effect. There is a need to better understand the possible mechanisms that mediate intervention effects in RCTs of implementation interventions to gain insight into how effective interventions change behaviour and why ineffective interventions do not. A challenge for implementation researchers is to develop methods for exploring these causal mechanisms alongside rigorous tests of implementation interventions.

There is increasing recognition of the value of process evaluations alongside trials of complex interventions such as professional behaviour change interventions [12-14]. Process evaluations complement outcome evaluation by investigating how an intervention may work; how it is delivered, the mechanisms through which effects may operate and its contextual moderators [12]. Process evaluations can offer robust explanations of why an intervention fails to improve health care (or even does harm) by assessing whether or not the intervention changes the proposed mediators of improved outcomes. Process evaluations often involve the ad hoc selection of context-specific indicators of process and use quantitative and/or qualitative methods to provide a detailed assessment of processes rooted in the context of the trial. Rather than ad hoc selection of process indicators, selecting indicators informed by theories of behaviour is an arguably superior approach to understand the determinants of the outcome. In turn, this could increase the ability to generalise findings to other clinical problems, professional groups and settings.

Behavioural science has systematically operationalized theories concerning determinants of behaviour and how they are associated with each other. This may be useful for understanding the mechanisms underlying implementation interventions designed to change clinicians' behaviour [15]. Such theories employ standard definitions of constructs and measurement methods, which may be useful for exploring causal mechanisms of implementation 
interventions by testing whether intervention effects operate via hypothesised mediating pathways. Theory-based process evaluations can therefore contribute to the accumulation of a knowledge base of how implementation interventions might operate [16].

Using theory to explore mediating mechanisms of behaviour change interventions is commonplace in some fields $[17,18]$ and shows promise for greater use in exploring the mechanisms of action in implementation interventions where healthcare professional behaviour change is involved. For example, Ramsay and colleagues [19] conducted a post-intervention theory-based process evaluation of two interventions aiming to reduce inappropriate test-ordering evaluated within a randomised trial. The process evaluation focused upon investigating the causal mechanisms of the intervention for three of the targeted tests. They showed that behavioural intention partially mediated the intervention effect in two of the three tests assessed and suggested that the lack of mediation for the third test may have partly been an function of a ceiling effect on intention. Hrisos and colleagues conducted a theory-based process evaluation alongside an intervention designed to change physicians' intentions and found that a persuasive communications intervention was mediated by theory-based constructs (attitude and subjective norm) [20]. These examples demonstrate the utility of drawing upon behavioural theory to hypothesise and test the mediating mechanisms of interventions for promoting health professional behaviour change.

When interventions are explicitly theory-based, the selection of a particular theory upon which to base the process evaluation is straightforward and can explicitly tie the intervention to potential mediating pathways $[14,21]$. However, many implementation interventions are designed pragmatically without an explicit theoretical basis but likely involve an implicit model of how the intervention may change clinicians' behaviour [15]. Such implicit models can be to some extent reverseengineered by examining the description of the intervention content, which provides an indication of the factors the intervention designers assumed needed to change. Thus, with sufficient intervention description, implicit models can be mapped onto theoretical constructs that are likely to be changed $[22,23]$. Linking these constructs back to a theoretical model that includes such constructs provides a basis for assessing mediating mechanisms through which the intervention effects can be hypothesised to operate regardless of whether the intervention itself is explicitly theory-based.

The theory of planned behaviour [24] (TPB) is a social cognition model of behaviour with well evidenced predictive utility across a number of populations and behaviours [25-27]. Applied to clinicians' thiazide prescribing behaviour, the TPB proposes that the most proximal antecedents of whether a clinician will perform a behaviour (in this case, prescribing thiazides) are their intention to perform the behaviour (whether they want to prescribe thiazides) and their perceived behavioural control (PBC) over the behaviour (whether they believe that they can prescribe thiazides). Their intention is in turn determined by three underlying cognitive constructs: their attitude (i.e., are they in favour or against prescribing thiazides), their subjective norm (their views of whether others think they should prescribe thiazides) and their PBC (see Fig. 1). A systematic review showed that studies using the TPB explained $59 \%$ of the variance in intention and $35 \%$ of the variance in health professional behaviour [27]. Behavioural theory has also been used to evaluate the process of trials of implementation interventions [19, 20, 28, 29] suggesting that behavioural theory may contribute to building a cumulative understanding of why implementation interventions are successful or not.

PEMs are a mode of delivering information. An implicit model might suggest a direct relationship between knowledge and behaviour change; however, such a model is not consistent with evidence [30]. The relationship between providing information and achieving behaviour change is more complex. When information is evidence-based and presented by a credible and influential source, it may be persuasive and could plausibly improve clinicians' motivation to prescribe by altering their beliefs about consequences (e.g. attitude) and highlighting the social influences (e.g. subjective norm) of prescribing [23]. The TPB captures the motivational constructs that PEMs might plausibly influence [31]. Given the TPB's evidenced predictive validity [27], it was arguably an appropriate model for evaluating the process of behaviour change in the pragmatic intervention tested in the OPEM trial.

That said, the TPB is not without its limitations and critics, and there is debate in some literatures about whether to retire the theory altogether $[32,33]$. Furthermore, the TPB is not the only behavioural theory that could be selected to explain the process of behaviour change in implementation interventions [34]. Nevertheless, given the plausibility that PEMs may change behaviour through a motivational process, the TPB provides a replicable mediation model and measurement methods for understanding how behaviour change might operate through a motivational process in health professionals. Furthermore, using the TPB as a basis for process evaluation using an experimental design provides an opportunity to test the tenets of the theory itself, further contributing to the evidence to inform the utility of the theory moving forward. And irrespective of the theory itself, the TPB provides a potentially useful methodological 


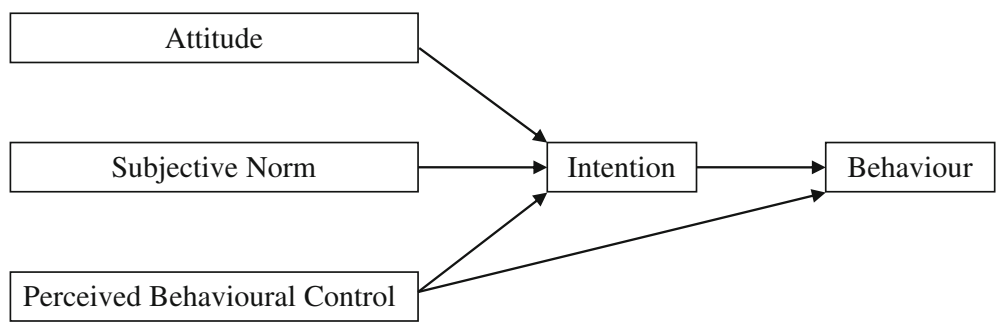

Fig. 1 The theory of planned behaviour [24]

exemplar for hypothesising and investigating mechanisms of action alongside trials of implementation interventions. We hypothesised that the OPEM intervention would be most likely to operate by changing physicians' intention to prescribe thiazides to elderly patients due to improved attitude or subjective norm with little or no change in perceived behavioural control [16].

\section{Methods}

\section{Summary of the main OPEM trial and OPEM TRY-ME} sub-trial intervention

The detailed methods of the OPEM trial, the OPEM TRYME sub-trial and this theory-based process evaluation are available in the published protocols [16, 31, 35]. In summary, the OPEM trial was replicated three times for three different areas of quality improvement, and we conducted independent process evaluations of two of these replications of the OPEM trial. Results of the other replicate process evaluation are reported elsewhere [36]. The current paper describes the results of the OPEM trial testing PEMs aimed at increasing physicians' prescription of thiazide diuretics for managing uncomplicated hypertension in patients $\geq 65$ years old in Ontario, Canada.

Family physicians (FPs) in the OPEM trial were randomised to receive PEMs of differing levels of detail along with informed, an evidence-based newsletter mailed quarterly to approximately 15,000 Ontario FPs since 1994.

The OPEM trial team developed two forms of the PEMs, and a team of health psychologists independently developed a third version of the PEMs (the latter featuring in the OPEM TRY-ME sub-trial). The OPEM trial team developed long educational messages produced as a two-page insert into informed (indistinguishable from the rest of the periodical in size, style and editing) including background and evidence-based guideline and references. The OPEM trial team also developed short directive statements produced on a postcard-sized card stapled to the outside of informed as 'outserts', which were composed of brief information about the benefits of thiazides compared to other antihypertensive drugs. The trial team developed these outserts pragmatically (atheoretically) with a focus on clear communication of an actionable message, rather than a specific theory. In addition to these two PEMs, an independent team of health psychologists developed a third form of PEM: TPB-based outserts designed to address intention, attitude and subjective norm [31]. The 'TPB outserts' were similar in format (length, size, design) to the atheoretical outserts.

Using a $2 \times 3$ factorial design, FPs were randomised to receive either an atheoretical outsert only, a TPB outsert only, a long insert only, a long insert and atheoretical outsert, a long insert and TPB outsert only or a copy of the informed newsletter with no message (control condition).

The main OPEM trial results are reported elsewhere [11]. The current paper reports results of the process evaluation for the $2 \times 3$ factorial test of the effects of long inserts, short atheoretical outserts and short TPB outserts on changes in the TPB constructs (attitude, subjective norm and intention) that we hypothesised would explain change exerted by the intervention.

\section{Theory-based process evaluation study participants and sample size}

Sampling and postal survey delivery proceeded as described in the protocol [16]. The OPEM trial team provided a sampling frame based on a random subset of physicians in the trial, excluding those sampled for the first process evaluation. We required 252 participants (63 per group) to have $80 \%$ power of detecting an effect size of 0.5 standard deviations using a significance level of $5 \%$. We assumed a $50 \%$ response rate for each survey (pre- and post-intervention), so our initial sample size was 1512 participants (252 per group).

\section{Questionnaire development}

We developed questionnaires in accordance with standard methods [37] and the protocol [16]. We included a contextualising patient scenario, which preceded the TPB items and described a representative set of clinical and contextual features in which the targeted prescribing behaviour would typically take place, describing a patient 
with recurrent elevated blood pressure readings and unsuccessful attempts at controlling blood pressure with lifestyle modifications (see Additional file 1 for the scenario). The questionnaire included 18 items directly measuring TPB constructs scored on a seven-point Likert scale (see Additional file 1). The items were presented in random order except for attitude items, which were ordered consecutively to distinguish this construct's response format (varying bipolar anchors) from the other TPB construct items. Each item was constructed using the Target, Action, Context and Time (TACT) principle [38] to specify the clinical behaviour under investigation. The Target was 'this woman' (referring to a description provided in the contextualising scenario); 'prescribing thiazide diuretics' was the Action; the Context was the 4th visit since her last annual physical (described in the scenario); and Time was (implicitly) during the consultation described in the scenario.

We supplemented TPB measures with context-specific items and self-reported past behaviour. A final openended question asked physicians in your experience, what are the reasons (medical and non-medical) for which elderly patients may not be prescribed thiazide diuretics as a first-line treatment for their hypertension?'

\section{Data collection procedure}

At baseline, we mailed the questionnaire using Dillman's tailored design method for postal questionnaires [39]. We restricted the questionnaire to two pages in length and provided $\mathrm{CDN} \$ 20$ to every physician who returned a completed questionnaire. We sent preintervention questionnaires 8 weeks prior to disseminating the PEMs and post-intervention questionnaires to baseline respondents 6 months after dissemination of the PEMs.

TPB items were reverse-scored (where necessary) such that high scores represented agreement (or positive attitudes) and low scores, disagreement (or negative attitudes). We used the item mean scores within each theory-based construct (i.e. intention, attitude, subjective norm, and perceived behavioural control) to create a composite score for each construct. Three independent research assistants verified the accuracy of the data entry.

\section{Analyses}

We assessed internal consistency (Cronbach's alpha) on TPB constructs before creating mean scores for each construct. We used analyses of covariance to test the hypotheses and used a $p$ value of 0.01 to adjust for multiple testing. We used a non-parametric analysis (Kruskal-Wallis test) to see whether results were robust against violations of assumptions. Low levels of missing data $(<7 \%)$ on TPB variables suggested that listwise deletion to handle missing data would be appropriate. We tested the appropriateness of the missing data strategy by re-running the analyses using two modified versions of the data: one which replaced all missing TPB values with data most supportive of our hypotheses and another replacing with data most opposed to our hypotheses.

We extracted demographic data from a physician database for a random sample of $20 \%$ of respondents and non-respondents to the pre-intervention questionnaire to test for non-response bias. We used ANOVAs to assess the impact of attrition by comparing preintervention TPB of completers of pre- and postintervention questionnaires to those who only completed pre-intervention surveys.

We conducted regression analyses with control group data to test the predictive utility and predictive pathways in the model. We regressed intention on attitude, subjective norm and perceived control (PBC) at baseline and regressed Time 2 self-reported behaviour on baseline intention and $\mathrm{PBC}$.

Two researchers (JP, NR) coded all comments from the open-ended question (about perceived barriers that may prevent an elderly patient from being prescribed thiazides) such that two people coded each comment. Coding was compared and differences resolved through discussion, and then codes were grouped into themes reflecting the perceived source of each barrier.

\section{Results}

\section{Response rate and non-response analysis}

Six hundred and thirty-two (of 1512; $41.8 \%$ ) physicians returned the pre-intervention questionnaire, 468 (74.1\% of baseline, $31.0 \%$ of total invited) of which also returned the post-intervention questionnaire (Table 1). Responder analysis showed that respondents were more likely to be affiliated with a university (9.2 vs. $2.1 \%, p<0.01)$ and more likely to be members of the College of Family Physicians of Canada (CFPC) (43.7 vs. $30.8 \%, p<0.05$ ) than non-respondents (see Table 2). No differences were observed on baseline TPB constructs between those completing both time-points compared to those only completing baseline. Eligibility for inclusion in all subsequent analyses was defined as all respondents completing both pre- and post-intervention surveys with no missing data on any TPB variables $(N=426)$.

\section{Internal consistency}

The Cronbach's alpha for intention was 0.96 and 0.95 for pre- and post-intervention questionnaires (respectively), for subjective norm $\alpha=0.90$ and for attitude $\alpha=0.86$ for both pre- and post-intervention, and for PBC, $\alpha=0.84$ (pre-intervention) and 0.82 (post-intervention). 
Table 1 Participant flow by group

\begin{tabular}{|c|c|c|c|c|c|c|}
\hline & \multicolumn{6}{|l|}{ Groups } \\
\hline & Insert only & Atheoretical outsert & TPB outsert & Insert and atheoretical outsert & Insert and TPB outsert & Control \\
\hline Allocated and invited & 252 & 252 & 252 & 252 & 252 & 252 \\
\hline Baseline return & 96 & 104 & 98 & 110 & 106 & 118 \\
\hline Follow-up return & 65 & 76 & 76 & 86 & 76 & 89 \\
\hline Excluded listwise (missing data) & 9 & 4 & 6 & 6 & 8 & 9 \\
\hline Included in analysis & 56 & 72 & 70 & 80 & 68 & 80 \\
\hline
\end{tabular}

\section{Main effects of PEMs on intention, attitude, subjective norm and PBC}

Physicians reported strong baseline intention to prescribe thiazides (overall mean $=5.93$ out of $7 ; \mathrm{SD}=1.44$; see Table 3 for details for each group). Controlling for pre-intervention intention, we did not observe a main effect for long insert, pragmatic outsert or TPB outsert PEMs on post-intervention intention to prescribe thiazides (primary outcome; see Table 4). On pre-intervention measures, physicians reported positive attitude (overall mean $=5.80, S D=1.09)$, strong agreement with stated subjective norm (overall mean $=5.83, \mathrm{SD}=1.06$ ) and strong agreement with statements of perceived behavioural control (overall mean $=6.20, \mathrm{SD}=1.01$ ); see Table 2 for between group details. No significant main effects were observed for insert, pragmatic outsert or TPB outsert PEM groups on any of the three theory-based predictors of behavioural intention (secondary outcomes; see Table 5). The Kruskal-Wallis test also reflected these findings, as did analyses testing the appropriateness of listwise deletion as a missing data strategy. These null findings indicated that further mediation analyses as originally described in the protocol would be inappropriate as the first criterion for mediation was not met given that the intervention did not alter the theory-based mediators.

\section{Testing the predictive efficacy of the TPB within the control group}

In the control group, ${ }^{1}$ baseline attitude $(r=0.72, p<0.01)$, subjective norm $(r=0.79, p<0.01)$, and perceived behavioural control $(r=0.81, p<0.01)$ were all strongly correlated with baseline intention. Baseline intention $(r=0.66, p<0.01)$ and perceived behavioural control ( $r=0.55, p<0.01$ ) both strongly correlated with follow-up self-reported behaviour. Residuals were plotted and were sufficiently normally distributed to proceed with interpretation. Regression of baseline intention scores on baseline TPB predictors of intention showed that attitude $(\beta=0.28$, $p<0.01, B=0.41,95 \% \mathrm{CI}_{B} 0.19$ to 0.64$)$, subjective norm $\left(\beta=0.28, p<0.05, B=0.38,95 \% \mathrm{CI}_{B} 0.09\right.$ to 0.68$)$ and perceived behavioural control scores $(\beta=0.40, p<0.01$, $B=0.5395 \% \mathrm{CI}_{B} 0.25$ to 0.81$)$ all significantly contributed to explaining $73.9 \%$ of the variance $\left(R_{\text {adj }}^{2}\right)$ in baseline intention. Baseline intention $(\beta=0.63, p<0.01$, $B=1.27,95 \% \mathrm{CI}_{B} 0.67$ to 1.86$)$ but not $\mathrm{PBC}(\beta=0.04$, $p=0.78, B=0.11,95 \% \mathrm{CI}_{B}=-0.68$ to 0.90$)$ predicted Time 2 self-reported behaviour, accounting for $42.6 \%$ of its variance $\left(R_{\text {adj }}^{2}\right)$.

\section{Self-reported past behaviour}

Physicians at baseline self-reported prescribing thiazides to a mean of $6.63(S D=2.71)$ of their 10 most recently seen elderly patients newly diagnosed with uncomplicated hypertension, suggesting potential scope for improvement and for greater consistency between physicians.

\section{Content analysis of perceived barriers to prescribing thiazides to elderly patients}

Most physicians (95\% at baseline and $91 \%$ at follow-up) provided at least one reason describing why elderly patients may not be prescribed thiazides as a first-line treatment for hypertension. We coded

Table 2 Demographics comparison of baseline respondents and non-respondents

\begin{tabular}{llllll}
\hline Demographic factor & & Respondents & Non-respondents & Population $^{\text {a }}$ & Test results $^{\mathrm{b}}$ \\
\hline Graduating year & Mean & 1978 & 1977 & 1981 & $t(263)=0.329, p=0.742$ \\
Sex & Male & $77.3 \%$ & $78.8 \%$ & $63.0 \%$ & $X^{2}(1, N=265)=0.081, p=0.776$ \\
Urban/rural & Urban & $89.1 \%$ & $91.1 \%$ & $91.9 \%$ & $X^{2}(1, N=265)=0.302, p=0.582$ \\
University affiliation & Yes & $9.2 \%$ & $2.1 \%$ & $9.3 \%$ & $X^{2}(1, N=265)=6.771, p=0.009$ \\
CFPC member & Yes & $43.7 \%$ & $30.8 \%$ & $46.7 \%$ & $X^{2}(1, N=265)=4.684, p=0.030$ \\
\hline
\end{tabular}

$20 \%$ random sample. $n=119$ for respondents and $n=146$ for non-respondents (four (respondents) and six (non-respondents) participants could not be found using MD Select and are thus not included in the analysis)

apopulation based upon all physicians in Ontario specialising in either family medicine or physician/general practice $(N=10,429)$

${ }^{\mathrm{b}}$ Test results compare respondents to non-respondents 
Table 3 Descriptive statistics for theory of planned behaviour constructs by group, before and after the OPEM trial

\begin{tabular}{|c|c|c|c|c|c|c|c|c|c|c|}
\hline & & \multirow[b]{3}{*}{$N$} & \multirow{2}{*}{\multicolumn{2}{|c|}{$\begin{array}{l}\text { Intention } \\
\text { Mean (sd) }\end{array}$}} & \multirow{2}{*}{\multicolumn{2}{|c|}{$\begin{array}{l}\text { Attitude } \\
\text { Mean (sd) }\end{array}$}} & \multirow{2}{*}{\multicolumn{2}{|c|}{$\begin{array}{l}\text { Subjective norm } \\
\text { Mean (sd) }\end{array}$}} & \multirow{2}{*}{\multicolumn{2}{|c|}{$\begin{array}{l}\text { Perceived behavioural control } \\
\text { Mean (sd) }\end{array}$}} \\
\hline & & & & & & & & & & \\
\hline \multicolumn{2}{|l|}{ Groups } & & Baseline & Follow-up & Baseline & Follow-up & Baseline & Follow-up & Baseline & Follow-up \\
\hline \multirow[t]{3}{*}{ Insert } & Atheoretical outsert & 80 & $6.05(1.38)$ & $6.12(1.19)$ & $5.79(0.98)$ & $5.89(0.96)$ & $5.82(1.11)$ & $5.96(0.93)$ & $6.27(0.91)$ & $6.38(0.83)$ \\
\hline & TPB outsert & 68 & $5.86(1.62)$ & $5.78(1.57)$ & $5.75(1.16)$ & 5.65 (1.19) & $5.93(1.02)$ & $6.02(0.97)$ & $6.33(0.93)$ & $6.32(0.99)$ \\
\hline & No outsert & 56 & $6.07(1.29)$ & $5.86(1.46)$ & $5.84(1.11)$ & $5.90(1.12)$ & $5.90(1.00)$ & $5.70(1.12)$ & $6.26(0.90)$ & $6.14(1.14)$ \\
\hline \multirow[t]{3}{*}{ No insert } & Atheoretical outsert & 72 & $5.82(1.51)$ & $5.71(1.53)$ & $5.73(1.15)$ & $5.66(1.12)$ & $5.68(1.02)$ & $5.62(1.15)$ & $6.05(1.09)$ & $5.98(1.09)$ \\
\hline & TPB outsert & 70 & $5.86(1.43)$ & $5.76(1.58)$ & $5.76(1.18)$ & $5.80(1.14)$ & $5.87(1.10)$ & $5.69(1.17)$ & $6.18(1.07)$ & $6.19(1.08)$ \\
\hline & No outsert & 80 & $5.92(1.44)$ & $5.84(1.59)$ & $5.90(0.99)$ & $5.76(1.17)$ & $5.79(1.08)$ & $5.70(1.18)$ & $6.13(1.13)$ & $6.16(1.03)$ \\
\hline
\end{tabular}

TPB theory of planned behaviour

physicians' responses and organised codes into themes representing the source of perceived barriers. 'Patient clinical characteristics' ('Co-morbid conditions where other antihypertensives are better,' 'Allergy to sulfonamides or thiazides') were mentioned by most respondents to this question (75.7 \% baseline, $73.4 \%$ follow-up), followed by 'patient comfort and side effects' ('Electrolyte disturbances; weak + dizzy + confusion', 'Increased urinary frequency as side effect'), mentioned by $62 \%$ of physicians at baseline and $59.9 \%$ at follow-up. $11.8 \%$ of physicians at baseline and $10.6 \%$ at follow-up described their 'beliefs as not in favour of thiazides' ('ACE Inhibitors offer benefits above and beyond BP control and are often a better 1st line choice"). A smaller percentage of physicians described other barriers, including 'patient preference/adherence' ('Patient preference'), 'system-related' ('Perception that diuretics are old and outdated drugs', 'Pharmaceutical pressure for more expensive drugs'), physician 'beliefs in favour of thiazides' ('For this woman (Mrs. Kelly), I wouldn't have many reasons not to prescribe a thiazide diuretic') and 'other' (i.e. unique comments not fitting into other codes).

\section{Discussion}

This study tested whether an information-provision intervention designed to promote guideline recommended

Table 4 Results of analysis of covariance for primary outcome of change in intention $(N=426)$

\begin{tabular}{llllllll}
\hline Effects & $F$ & $p$ & $B$ & SE & $\frac{95 \% \mathrm{Cl}}{$\cline { 5 - 7 }} \\
\hline $\begin{array}{l}\text { Covariate } \\
\quad \text { Baseline Intention }\end{array}$ & 231.37 & $<0.01$ & 0.61 & 0.04 & 0.53 & 0.69 \\
$\begin{array}{l}\text { Main effects } \\
\text { Insert PEM }\end{array}$ & & & & & & \\
$\quad \begin{array}{l}\text { Outsert PEM (atheoretical) } \\
\text { Outsert PEM (TPB) }\end{array}$ & 0.42 & 0.52 & 0.09 & 0.14 & -0.19 & 0.37 \\
\hline
\end{tabular}

PEM printed educational materials, TPB theory of planned behaviour prescribing of thiazides to elderly patients with uncomplicated hypertension operated by modifying family physicians' attitudes, norms, control, and motivation to prescribe. The main OPEM trial itself did not observe any changes in thiazide prescription [11], and the intervention did not change theory-based determinants of thiazide prescription in this process evaluation. Nevertheless, the theoretical basis of this process evaluation provides a viable foundation for interpreting the trial's null findings, which may inform and help to optimise future interventions. This process evaluation contributes to and builds on the growing literature on theory-based process evaluation $[19,20,28,29]$ by developing methods, employing them alongside the OPEM trial, and hypothesising a priori the process through which trial effects would operate.

At baseline, the sample of physicians reported strong intention (they wanted to prescribe thiazides), positive attitude (they believed prescribing thiazides is a good idea), high subjective norm (people important to them thought they should prescribe thiazides), and strong PBC (they believed that they could prescribe thiazides) over prescribing thiazides. Medium/large changes in intention are often reflected in small/medium changes in behaviour [40]; given how high the reported intention was in the process evaluation in all trial arms, we would expect that a ceiling effect precludes even a medium effect in the main trial. The theoretical basis suggests that PEMs were ineffective because they aimed to educate and persuade physicians about performing a behaviour that they already strongly intended to do.

In open-ended questions, family physicians highlighted patient-related clinical complications as reasons why elderly patients may not be prescribed thiazides, reflecting beliefs about negative consequences associated with prescribing to patients with complications or side effects. However, attitude towards prescribing was positive, suggesting these negative beliefs did not represent overall attitude towards prescribing thiazides and therefore may not be influencing motivation and behaviour. Such findings 
Table 5 Results of analysis of covariance for secondary outcomes (change in attitude, subjective norm, and perceived behavioural control) $(N=426)$

\begin{tabular}{|c|c|c|c|c|c|c|c|}
\hline \multirow[t]{2}{*}{ TPB construct } & \multirow[t]{2}{*}{ Effects } & \multirow[t]{2}{*}{$F$} & \multirow[t]{2}{*}{$p$} & \multirow[t]{2}{*}{ B } & \multirow[t]{2}{*}{ SE } & \multicolumn{2}{|l|}{$95 \% \mathrm{Cl}$} \\
\hline & & & & & & Lower & Upper \\
\hline \multirow[t]{6}{*}{ Attitude } & Covariate & & & & & & \\
\hline & Baseline attitude & 184.72 & $<0.01$ & 0.61 & 0.04 & 0.53 & 0.69 \\
\hline & Main effects & & & & & & \\
\hline & Insert PEM & 0.82 & 0.37 & 0.08 & 0.09 & -0.09 & 0.25 \\
\hline & Outsert PEM (athoretical) & 0.06 & 0.82 & 0.03 & 0.11 & -0.19 & 0.24 \\
\hline & Outsert PEM (TPB) & 0.03 & 0.86 & -0.02 & 0.11 & -0.23 & 0.20 \\
\hline \multirow[t]{6}{*}{ Subjective norm } & Covariate & & & & & & \\
\hline & Baseline subjective norm & 80.10 & $<0.01$ & 0.57 & 0.04 & 0.49 & 0.65 \\
\hline & Main effects & & & & & & \\
\hline & Insert PEM & 3.69 & 0.06 & 0.17 & 0.09 & -0.004 & 0.34 \\
\hline & Outsert PEM (athoretical) & 1.45 & 0.23 & 0.13 & 0.11 & -0.08 & 0.34 \\
\hline & Outsert PEM (TPB) & 0.86 & 0.35 & 0.10 & 0.11 & -0.11 & 0.32 \\
\hline \multirow[t]{6}{*}{ Perceived behavioural control (PBC) } & Covariate & & & & & & \\
\hline & Baseline PBC & 127.08 & $<0.01$ & 0.49 & 0.04 & 0.40 & 0.57 \\
\hline & Main effects & & & & & & \\
\hline & Insert PEM & 1.20 & 0.27 & 0.10 & 0.09 & -0.08 & 0.27 \\
\hline & Outsert PEM (athoretical) & 0.11 & 0.74 & 0.04 & 0.11 & -0.17 & 0.25 \\
\hline & Outsert PEM (TPB) & 0.37 & 0.55 & 0.07 & 0.11 & -0.15 & 0.28 \\
\hline
\end{tabular}

nevertheless suggest that future intervention to improve thiazide prescribing could involve patients' views and behaviour as well.

Despite strong intention to prescribe, self-reported (6.63 of last 10 patients) and observed (28\% in main trial data) [11] thiazide prescription rates at baseline and follow-up did not reflect guideline recommendations. While prescription to every elderly patient with newly diagnosed uncomplicated hypertension may not always be appropriate and self-reported rates are likely an overestimation of actual prescribing rates of thiazides, there nevertheless remains considerable room for improving thiazide prescription. This also suggests that while intention played a necessary role in prescribing thiazides, additional factors may have moderated the translation of these strong intentions into higher prescription rates. The TPB focuses largely on the predictors of intention ('pre-intentional' factors) but does not include any postintentional factors to explain how intention is translated into action. This may have implications for interventions that only include techniques designed to increase motivation: had the OPEM interventions increased physicians' intention to prescribe thiazides, post-intentional barriers may still have limited actual behaviour change had it been observed.

This process evaluation provides an inherent test of the TPB in two ways: predictive utility and explanation of behaviour change. We showed that the TPB's predictive efficacy was consistent with systematic review evidence [27]. However, we could not assess whether the TPB explains behaviour change, as there was none to explain. Support for the TPB would require both a change in behaviour and a change in one or more TPB constructs, while evidence against the TPB would require a change in behaviour without any associated change in TPB constructs [16]; neither were possible here. Nevertheless, whether the TPB sufficiently captures all possible routes to behaviour change that a PEM may provide is debatable. There have been increasing calls from the literature to consider theories of behaviour that move beyond the TPB towards focusing on (in addition to attitudes, subjective norm, PBC and intention) post-intentional factors, habit, and automaticity [41] and the role of team and organisational factors $[42,43]$.

\section{Lessons learned and recommendations for conducting theory-based process evaluations}

This process evaluation may help to advance methods of conducting theory-based process evaluations alongside RCTs of implementation interventions to help to understand the mechanism of effect (or lack thereof). We offer the following recommendations based on our experience. Theory-based process evaluations conducted 
alongside randomised controlled trials should in as far as is possible:

a. Reflect the trial's design, including comparison and control group(s): For the OPEM process evaluation, this involved surveying a random sample of physicians from each of the main trial's arms to meet the sample size requirements.

b. Collect data from trial participants pre- and postintervention to control for baseline differences: We surveyed physicians at two time points, before and 6 months after the intervention, in all trial arms. We showed this to be feasible, and the pretest data demonstrated that there was little room for improvement in process measures from the start, which would not have been possible using a post-test only design.

c. Use previously tested theoretical models to provide consistency with the literature, generalizability, and foster a cumulative knowledge base: A key purpose of a scientific theory is to summarise existing knowledge. By selecting an established theory with constructs that could plausibly explain the mechanism of action of an education-focused intervention, the findings can be compared against the existing literature, thereby facilitating future evidence syntheses and informing future PEM-based interventions.

d. Hypothesise the mediating mechanism a priori and conduct a mediation analysis if effects detected on primary and/or secondary trial outcomes: We demonstrated how drawing upon a theory of behaviour provides the basis for hypothesising how the intervention's mechanism of action might operate through this model. The challenge, particularly for interventions developed without a theory, remains in selecting and operationalizing a plausible theory for explaining the intervention effect. Whether the most plausible theory was selected in the present study is not clear given the lack of change in either process or outcome measures. Nevertheless, selecting a theoretical model that proposes a mediation pathway provides a basis for testing its mechanisms of action when possible [19].

e. Conduct formative theory-based investigations of determinants of the targeted behaviour prior to the trial: The present study was conducted opportunistically alongside a pragmatic trial, and the findings helped to clarify why the intervention as specified was not effective in increasing thiazide prescribing. Ideally, the content of the PEMs might have been informed by formative research to investigate potential barriers and enablers [44]. While such approaches were not commonplace at the time the trial was conducted, future PEMbased interventions would benefit from assessing and addressing (in as much as is possible within the PEM-based method of delivery) the factors that may prevent change. Such formative investigations can also inform theory selection and early phase questionnaire research to ensure that there is 'room for improvement' in the anticipated mechanism of change prior to the trial and its process evaluation.

f. Assess the fidelity with which the intervention has been received, read, and responded to by the target participant: In the present study, we could not assess the extent to which recipients read the PEMs, which could be an effect modifier. Building in fidelity assessment alongside mechanistic process evaluation is advised when possible.

This process evaluation provides a theoretical basis upon which future implementation interventions targeting increased thiazide prescription could draw instead of 'going back to the drawing board'. In wellinformed and motivated health professionals, if PEMs only deliver content targeting motivation, this may not be sufficiently potent. Instead, methods of delivering techniques that address post-intentional barriers to prescribing thiazides may be preferred. In such instances, theories of behaviour change such as the health action process approach [45] and dual process models $[41,46]$ that include and go beyond motivation would be more informative than motivational models such as the TPB.

A strength of this study is its use of a well-tested theory of behaviour operationalized according to best recommended practice to investigate the underlying mechanisms of an implementation intervention. By matching the $2 \times 3$ factorial design of the OPEM trial and assessing TPB cognitions before and after the intervention was delivered, we quantified existing levels of attitude, subjective norm, perceived behavioural control, and intention, providing an explanation for the lack of change in behaviour observed in the OPEM trial. While we showed strong internal consistency on all measures, some social desirability bias may have led to over reporting. However, this seems unlikely given the relatively low self-reported behaviour (mean six of last ten patients) and confidential nature of the data collection. Future research would benefit from assessing social desirability bias in questionnaires to health professionals. This study is also limited by an inability to link theory-based constructs to objective measures of behaviour from the main trial. This process evaluation is also limited by an observed response bias towards physicians who are part of a university and part of the College of Family 
Physicians of Canada, who may have had greater access and exposure to evidence prior to the OPEM trial and may have had more positive attitude and intention relative to the full trial sample. Our response rate was a further limitation, despite our evidence-based efforts to maximise recruitment to this study. However, the response rate was similar to other theory-based studies with health professionals and underscores the recognised challenge of recruitment and retention of health professionals in such studies. Our qualitative analyses helped to supplement our quantitative findings with additional contextual insight. Future theory-based process evaluations could be well served to also assess such contextual factors quantitatively to investigate whether they may operate as moderators of the intervention alongside the mediated mechanisms of change.

\section{Conclusions}

By conducting a pre-post theory-based process evaluation matched to the factorial design of the main trial, this study advances the methodology of conducting process evaluations alongside randomised trials of implementation interventions and demonstrates the potential utility of drawing upon theory for interpreting the results of pragmatic trials. In this case, pre-existing strong intention, subjective norm, and positive attitude provide a theory-based explanation of why dissemination of printed educational materials may not result in a change in physicians' prescribing behaviour. The use of printed educational materials for increasing prescription rates may therefore be ineffective when physicians' preexisting motivation to prescribe is strong. Future efforts at increasing prescription rates should consider targeting post-intentional factors.

\section{Endnote}

${ }^{1} n=76$ analysed, four participants excluded due to missing data on follow-up self-reported behaviour

\section{Additional file}

Additional file 1: Electronic version of questionnaire. (PDF 89 kb)

\section{Acknowledgements}

The OPEM process evaluation study was developed as part of the CIHR funded interdisciplinary capacity enhancement team KT-ICEBeRG. Justin Presseau was funded by KT-ICEBeRG. Jeremy Grimshaw and Gaston Godin hold Canada Research Chairs. Louise Lemyre holds an R.S. McLaughlin Research Chair. We would like to thank Keith O'Rourke for his contribution to the analysis plan for the study. Ian Graham and Jeremy Grimshaw hold a CIHR Foundation Grant (FDN 143237 and FDN 333392, respectively).

\section{Funding}

The funder, Canadian Institutes of Health Research (CIHR FRS KTS-73423), was not involved in the design of the study, data collection, analysis, and interpretation of data or in writing the manuscript.
Availability of data and materials

Data is available from the corresponding author upon request. The questionnaire used in this study is available as an Additional file 1.

\section{Authors' contributions}

JP and JMG co-wrote the manuscript. JMG and MZ were co-Pls on the study. $J P$, JT, and NR contributed to the analyses. All authors contributed to the development of this study and interpretation of findings. All authors read and approved the final manuscript.

\section{Competing interests}

Martin Eccles is the founding Editor in Chief of Implementation Science; Justin Presseau, Jeremy Grimshaw, France Légaré, and lan Graham are members of the editorial board of Implementation Science. No authors were involved in editorial decision-making.

Consent for publication

Not applicable.

Ethics approval and consent to participate

This study has received approval from the Research Ethics Board at The Ottawa Hospital. Returned questionnaires were taken as consent to participate.

\section{Author details}

'Ottawa Hospital Research Institute, The Ottawa Hospital, General Campus, 501 Smyth Road, Box 201B, Ottawa, Ontario K1H 8L6, Canada. ${ }^{2}$ School of Epidemiology, Public Health and Preventive Medicine, 451 Smyth Road, Ottawa, Ontario K1H 8M5, Canada. ${ }^{3}$ Department of Medicine, University of Ottawa, 451 Smyth Rd, Ottawa, Ontario K1H 8M5, Canada. ${ }^{4}$ Retired, Ottawa, Canada. ${ }^{5}$ Institute of Health and Society, Newcastle University, Baddiley-Clark Building, Richardson Road, Newcastle Upon Tyne NE2 4AX, England. ${ }^{6}$ School of Health Sciences, City University London, Northampton Square, London EC1V OHB, UK. ${ }^{7}$ Faculty of Nursing, Laval University, Pavillon

Ferdinand-Vandry, 1050 Avenue de la Medicine, Room 1445, Quebec City, Quebec G1V 0A6, Canada. ${ }^{8}$ School of Nursing, Faculty of Health Sciences, University of Ottawa, 451 Smyth Rd, Ottawa, Ontario K1H 8M5, Canada.

${ }^{9}$ Canadian Diabetes Association, 522 University Ave, Toronto, ON M5G 2A2, Canada. ${ }^{10}$ Institute of Applied Health Sciences, College of Life Sciences and Medicine, 2nd floor, Health Sciences Building, Foresterhill, Aberdeen AB25 2ZD, UK. ${ }^{11}$ Department of Family Medicine and Emergency Medicine, Université Laval, Québec City, Québec G1K 7P4, Canada. ${ }^{12}$ School of Psychology, University of Ottawa, 120 University, Social Sciences Building FSS-5052, Ottawa, Ontario K1N 6N5, Canada. ${ }^{13}$ Centre for Studies in Family Medicine, Department of Family Medicine, Schulich School of Medicine and Dentistry, Western University, 1465 Richmond Street, London, Ontario N6A 3K7, Canada. ${ }^{14}$ Institute for Clinical Evaluative Sciences, University of Toronto, 2075 Bayview Avenue, Toronto, Ontario M4N 3M5, Canada.

Received: 2 December 2015 Accepted: 26 August 2016

Published online: 13 September 2016

\section{References}

1. Leenen $\mathbf{F H H}$, Dumais J, McInnis NH, Turton P, Stratychuk L, Nemeth $\mathrm{K}$, et al. Results of the Ontario survey on the prevalence and control of hypertension. Can Med Assoc J. 2008;178(11):1441-9.

2. Joffres M, Falaschetti E, Gillespie C, Robitaille C, Loustalot F, Poulter N, et al. Hypertension prevalence, awareness, treatment and control in national surveys from England, the USA and Canada, and correlation with stroke and ischaemic heart disease mortality: a cross-sectional study. BMJ Open. 2013;3(8):e003423.

3. Wright JM. Choosing a first-line drug in the management of elevated blood pressure: what is the evidence? 1: thiazide diuretics. CMAJ. 2000;163(1):57-60.

4. The ALLHAT Officers and Coordinators for the ALLHAT Collaborative Research Group. Major outcomes in high-risk hypertensive patients randomized to angiotensin-converting enzyme inhibitor or calcium channel blocker vs diuretic: the Antihypertensive and Lipid-Lowering Treatment to Prevent Heart Attack Trial (ALLHAT). JAMA J Am Med Assoc. 2002;288(23):2981-97. 
5. Canadian Hypertension Education Program. 2007 CHEP Recommendations for the management of hypertension [Internet]. 2007. Available from: http://www. hypertension.ca/ hyperten/images/stories/dls/chep-2007-spiral-mar16.pdf. Accessed 12 May 2016

6. Daskalopoulou SS, Rabi DM, Zarnke KB, Dasgupta K, Nerenberg K, Cloutier L, et al. The 2015 Canadian Hypertension Education Program recommendations for blood pressure measurement, diagnosis, assessment of risk, prevention, and treatment of hypertension. Can J Cardiol. 2015;31(5):549-68.

7. Fretheim A, Aaserud M, Oxman AD. The potential savings of using thiazides as the first choice antihypertensive drug: cost-minimisation analysis. Implement Sci. 2003;3:18.

8. Siegel D. Changes in the pharmacologic treatment of hypertension in the department of veterans affairs 1997-1999: decreased use of calcium antagonists and increased use of $\beta$-blockers and thiazide diuretics. Am J Hypertens. 2001;14(9):957-62.

9. Giguère A, Légaré $F$, Grimshaw J, Turcotte $S$, Fiander M, Grudniewicz A, et al. Printed educational materials: effects on professional practice and healthcare outcomes. In: The Cochrane Collaboration, editor. Cochrane Database of Systematic Reviews [Internet]. Chichester: John Wiley \& Sons, Ltd; 2012. [cited 2016 May 9]. Available from: http://doi.wiley.com/10.1002/ 14651858.CD004398.pub3.

10. Grudniewicz A, Kealy R, Rodseth RN, Hamid J, Rudoler D, Straus SE. What is the effectiveness of printed educational materials on primary care physician knowledge, behaviour, and patient outcomes: a systematic review and meta-analyses. Implement Sci. 2015;10(1):164.

11. Zwarenstein M, Grimshaw JM, Presseau J, Francis JJ, Godin G, Johnston M, et al. Printed educational messages fail to increase use of thiazides as firstline medication for hypertension in primary care: a cluster randomized controlled trial [ISRCTN72772651]. Implement Sci.

12. Moore GF, Audrey S, Barker M, Bond L, Bonell C, Hardeman W, et al. Process evaluation of complex interventions: Medical Research Council guidance. BMJ. 2015;350:h1258.

13. Oakley A. Process evaluation in randomised controlled trials of complex interventions. BMJ. 2006:332(7538):413-6.

14. Craig P, Dieppe P, Macintyre S, Michie S, Nazareth I, Petticrew M. Developing and evaluating complex interventions: the new Medical Research Council guidance. BMJ. 2008;337:a1655.

15. Eccles M, Grimshaw J, Walker A, Johnston M, Pitts N. Changing the behavior of healthcare professionals: the use of theory in promoting the uptake of research findings. J Clin Epidemiol. 2005;58(2):107-12.

16. Grimshaw JM, Zwarenstein M, Tetroe JM, Godin G, Graham ID, Lemyre L, et al. Looking inside the black box: a theory-based process evaluation alongside a randomised controlled trial of printed educational materials (the Ontario Printed Educational Message, OPEM) to improve referral and prescribing practices in primary care in Ontario Canada. Implement Sci. 2007;2(1):38.

17. Jones LW, Courneya KS, Fairey AS, Mackey JR. Does the theory of planned behavior mediate the effects of an oncologist's recommendation to exercise in newly diagnosed breast cancer survivors? Results from a randomized controlled trial. Health Psychol. 2005;24(2):189-97.

18. Hardeman W, Kinmonth A, Michie S, Sutton S, the ProActive Project Team. Impact of a physical activity intervention program on cognitive predictors of behaviour among adults at risk of Type 2 diabetes (ProActive randomised controlled trial). Int J Behav Nutr Phys Act. 2009;6(1):16.

19. Ramsay CR, Thomas RE, Croal BL, Grimshaw JM, Eccles MP. Using the theory of planned behaviour as a process evaluation tool in randomised trials of knowledge translation strategies: a case study from UK primary care. Implement Sci. 2010;5(1):71.

20. Hrisos S, Eccles M, Johnston M, Francis J, Kaner EF, Steen N, et al. An intervention modelling experiment to change GPs' intentions to implement evidence-based practice: using theory-based interventions to promote GP management of upper respiratory tract infection without prescribing antibiotics \#2. BMC Health Serv Res. 2008;8(1):10.

21. Michie S, Fixsen D, Grimshaw JM, Eccles MP. Specifying and reporting complex behaviour change interventions: the need for a scientific method. Implement Sci. 2009;4(1):40.

22. Little EA, Presseau J, Eccles MP. Understanding effects in reviews of implementation interventions using the Theoretical Domains Framework. Implement Sci. 2015;10(1):90.

23. Michie S, Johnston M, Francis J, Hardeman W, Eccles M. From theory to intervention: mapping theoretically derived behavioural determinants to behaviour change techniques. Appl Psychol. 2008;57(4):660-80.
24. Ajzen I. The theory of planned behavior. Organ Behav Hum Decis Process. 1991;50(2):179-211.

25. McEachan RRC, Conner M, Taylor NJ, Lawton RJ. Prospective prediction of health-related behaviours with the theory of planned behaviour: a metaanalysis. Health Psychol Rev. 2011;5(2):97-144.

26. Presseau J, Johnston M, Francis JJ, Hrisos S, Stamp E, Steen N, et al. Theorybased predictors of multiple clinician behaviors in the management of diabetes. J Behav Med. 2014;37(4):607-20.

27. Godin G, Bélanger-Gravel A, Eccles M, Grimshaw J. Healthcare professionals' intentions and behaviours: A systematic review of studies based on social cognitive theories. Implement Sci. 2008;3(1):36.

28. Francis JJ, Eccles MP, Johnston M, Whitty P, Grimshaw JM, Kaner EF, et al. Explaining the effects of an intervention designed to promote evidencebased diabetes care: a theory-based process evaluation of a pragmatic cluster randomised controlled trial. Implement Sci. 2008;3(1):50.

29. Watson MC, Walker A, Grimshaw JM, Bond CM. Why educational interventions are not always effective: a theory-based process evaluation of a randomised controlled trial to improve non-prescription medicine supply from community pharmacies. Int J Pharm Pract. 2006;14(4):249-54

30. Bonetti D, Pitts NB, Eccles M, Grimshaw J, Johnston M, Steen N, et al. Applying psychological theory to evidence-based clinical practice: identifying factors predictive of taking intra-oral radiographs. Soc Sci Med. 2006;63(7):1889-99.

31. Francis JJ, Grimshaw JM, Zwarenstein M, Eccles MP, Shiller S, Godin G, et al. Testing a TheoRY-inspired MEssage ('TRY-ME'): a sub-trial within the Ontario Printed Educational Message (OPEM) trial. Implement Sci. 2007;2(1):39.

32. Sniehotta FF, Presseau J, Araújo-Soares V. Time to retire the theory of planned behaviour. Health Psychol Rev. 2014;8(1):1-7.

33. Sniehotta FF, Presseau J, Araújo-Soares V. On the development, evaluation and evolution of health behaviour theory. Health Psychol Rev. 2015;9(2):176-89.

34. Michie $\mathrm{S}$, editor. $\mathrm{ABC}$ of behaviour change theories: [an essential resource for researchers, policy makers and practitioners; 83 theories]. London: Silverback Publ; 2014.

35. Zwarenstein M, Hux JE, Kelsall D, Paterson M, Grimshaw J, Davis D, et al. The Ontario Printed Educational Message (OPEM) trial to narrow the evidencepractice gap with respect to prescribing practices of general and family physicians: a cluster randomized controlled trial, targeting the care of individuals with diabetes and hypertension in Ontario Canada. Implement Sci. 2007;2(1):37.

36. Grimshaw JM, Presseau J, Tetroe J, Eccles MP, Francis JJ, Godin G, et al. Looking inside the black box: results of a theory-based process evaluation exploring the results of a randomized controlled trial of printed educational messages to increase primary care physicians' diabetic retinopathy referrals [Trial registration number ISRCTN72772651]. Implement Sci. 2014;9(1):86.

37. Francis JJ, Eccles MP, Johnston M, Walker A, Grimshaw J, Foy R, et al. Constructing questionnaires based on the theory of planned behaviour: a manual for health services researchers [Internet]. Centre for Health Services Research: University of Newcastle upon Tyne; 2004. Available from: http:// openaccess.city.ac.uk/1735/. Accessed 12 May 2016.

38. Fishbein $M$, editor. Readings in attitude theory and measurement. New York: Wiley; 1967. 499 p.

39. Dillman DA. The design and administration of mail surveys. Annu Rev Sociol. 1991;17:225-49.

40. Webb TL, Sheeran P. Does changing behavioral intentions engender behavior change? A meta-analysis of the experimental evidence. Psychol Bull. 2006;132(2):249-68.

41. Presseau J, Johnston M, Heponiemi T, Elovainio M, Francis JJ, Eccles MP, et al. Reflective and automatic processes in health care professional behaviour: a dual process model tested across multiple behaviours. Ann Behav Med. 2014;48(3):347-58.

42. Elovainio M, Steen N, Presseau J, Francis J, Hrisos S, Hawthorne G, et al. Is organizational justice associated with clinical performance in the care for patients with diabetes in primary care? Evidence from the improving Quality of care in Diabetes study. Fam Pract. 2013;30(1):31-9.

43. Eccles MP, Hrisos S, Francis JJ, Steen N, Bosch M, Johnston M. Can the collective intentions of individual professionals within healthcare teams predict the team's performance: developing methods and theory. Implement Sci. 2009;4(1):24.

44. French SD, Green SE, O'Connor DA, McKenzie JE, Francis JJ, Michie S, et al. Developing theory-informed behaviour change interventions to implement 
evidence into practice: a systematic approach using the theoretical domains framework. Implement Sci. 2012;7(1):38.

45. Schwarzer R. Modeling health behavior change: how to predict and modify the adoption and maintenance of health behaviors. Appl Psychol. 2008;57(1):1-29.

46. Strack F, Deutsch R. Reflective and impulsive determinants of social behavior. Personal Soc Psychol Rev. 2004;8(3):220-47.

Submit your next manuscript to BioMed Central and we will help you at every step:

- We accept pre-submission inquiries

- Our selector tool helps you to find the most relevant journal

- We provide round the clock customer support

- Convenient online submission

- Thorough peer review

- Inclusion in PubMed and all major indexing services

- Maximum visibility for your research

Submit your manuscript at www.biomedcentral.com/submit
Biomed Central 